\section{Immer wieder Tonsillitis: OP oder erstmal abwarten?}

\author{
Werden Kindern mit rezidivierenden Tonsillitiden die Rachenmandeln ent- \\ fernt, erkranken sie offenbar im darauffolgenden Jahr seltener an Rachen- \\ infektionen als ohne Operation.
}

$\mathrm{O}_{\mathrm{n}}^{\mathrm{b}}$ b die Tonsillektomie für Patienten mit rezidivierenden Tonsillitiden Vorteile gegenüber einer Watchful-Waiting-Strategie hat, haben Anna Morad vom Vanderbilt University Medical Center in Nashville, Tennessee, und Kollegen in einem aktuellen Review untersucht. Grundlage hierfür waren sieben Studien aus den Jahren 1980 bis 2016, in denen die beiden Verfahrensweisen verglichen wurden. Die Kinder, die in die Untersuchung eingeschlossen waren, hatten in den vorausgegangenen ein bis drei Jahren mindestens drei leichte bis mittelstarke Racheninfektionen durchgemacht.

In den ersten zwölf Monaten nach der Operation war ein Vorteil für die Kinder nach Tonsillektomie gegenüber den beobachteten bzw. konservativ behandelten Patienten erkennbar: Die operierten Kinder fehlten weniger häufig in der Schule, erkrankten seltener an Rachen- infektionen, hatten weniger Arztkontakte, und auch die Häufigkeit nachgewiesener A-Streptokokken-Infektionen war geringer.

Bei der Zahl der Racheninfektionen zeigten sich in verschiedenen Studien folgende Unterschiede im ersten Jahr nach der Tonsillektomie vs. Watchful Waiting: 0,5 vs. $0,64 / \mathrm{Jahr}$ bzw. 1,74 vs. 2,93 bzw. 0,56 vs. 0,77 . In einer retrospektiven Kohortenstudie wurde bei nicht operierten Kindern über dreimal so viele positive A-Streptokokken-Tests berichtet wie bei Kindern nach Tonsillektomie. In einer weiteren Kohortenstudie war die Zahl der Arztbesuche nach der Tonsillektomie gesunken und gegenüber der Beobachtungsgruppe in den ersten drei Jahren um jährlich 2,5 geringer. Nach vier bis sechs Jahren zeigte sich jedoch nur noch ein Unterschied von 1,4 Arztbesuchen jährlich. Im Hin- blick auf die Lebensqualität ergaben sich keine Unterschiede, sie verbesserte sich sowohl mit als auch ohne Tonsillektomie.

Der positive Effekt, den die OP im ersten Jahr brachte, hält offenbar nicht an. So konnte eine langfristige Verringerung von Racheninfektionen nach Tonsillektomie gegenüber den konservativ behandelten Patienten in den analysierten Untersuchungen nicht belegt werden. Waren nach Entfernung der Rachenmandeln mehr als zwölf Monate vergangen, zeigten sich zwischen den beiden Gruppen zudem weder Unterschiede bei der Häufigkeit von Streptokokkeninfektionen noch bei der Anzahl der Fehltage in der Schule.

Fazit: Die Fragen zum Langzeitnutzen einer Tonsillektomie bei rezidivierenden Tonsillitiden müssten künftige Studien mit höheren Probandenzahlen klären, in denen auch Schwere und Art der Racheninfektionen berücksichtigt werden sollten, resümieren Morad und Kollegen.

Dr. Christine Starostzik

Morad A, Sathe NA, Francis DO, et al. Tonsillectomy Versus Watchful Waiting for Recurrent Throat Infection: A Systematic Review. Pediatrics. 2017; 139 (2): e20163490

\section{Nasopharynxkarzinom: Man kann noch mehr tun}

\author{
Beim fortgeschrittenen, aber nicht metastasierten Nasopharynxkarzinom hat \\ sich die zeitgleiche Gabe von Strahlen- und Chemotherapie (CRT) etabliert. \\ Wäre eine zusätzliche adjuvante oder Induktions-CT hilfreich?
}

$B^{\mathrm{c}}$ eim lokal fortgeschrittenen Nasopharynxkarzinom hat sich in den vergangenen Jahren gezeigt, dass die zeitgleiche Gabe einer Chemo- und Radiotherapie Gesamtüberleben (OS), progressionsfreies Überleben (PFS), lokoregionäre Kontrolle und Fernmetastasenkontrolle verbessert. Kontrovers blieb, ob eine zusätzliche Induktions- oder adjuvante CT sinnvoll ist. In eine große Datenbank flossen Daten von 5.144 Patienten aus insgesamt 20 randomisierten Studien zur RT +/- CT beim nichtmetastasierten Nasopharynxkarzinom ein. Die Behandlungen wurden in sieben Kategorien eingeteilt:
- RT allein (RT),

_ Induktions-CT gefolgt von RT (IC-RT), — RT gefolgt von adjuvanter CT (RT-AC), - Induktions-CT gefolgt von RT gefolgt von adjuvanter CT (IC-RT-AC), _zeitgleiche CRT (CRT),

- Induktions-CT gefolgt von CRT (ICCRT),

—CRT gefolgt von adjuvanter CT (CRTAC).

Am wahrscheinlichsten wurde das OS verbessert durch CRT-AC, CRT und ICCRT. Die Hazard Ratios (HR) im Vergleich zur alleinigen RT betrugen 0,65, 0,77 bzw. 0,81. Beim Vergleich von CRT-
AC und CRT lag die HR für OS bei 0,85, für PFS bei 0,81, für lokoregionäre Kontrolle bei 0,70 und für Fernmetastasenkontrolle bei 0,87 . IC-CRT lag im Vergleich der verschiedenen Strategien auf dem 1. Platz für die Fernmetastasenkontrolle, auf dem 2. Platz für das PFS.

Fazit: Eine zusätzliche adjuvante CT nach CRT führte zum höchsten Überlebensvorteil der Patienten mit nicht metastasiertem Nasopharynxkarzinom. Die Addition einer Induktions-CT zur CRT hatte den besten Einfluss auf die Fernmetastasenkontrolle. Allerdings sollte bei der Interpretation indirekter Vergleiche immer Vorsicht geboten sein, so die Forscher.

Christina Berndt

Ribassin-Majed L et al. What Is the Best Treatment of Locally Advanced Nasopharyngeal Carcinoma? An Individual Patient Data Network Meta-Analysis. J Clin Oncol. 2016 Dec 5. [Epub ahead of print]. 